\title{
FIBRINOGÊNIO COMO FATOR DE RISCO INDEPENDENTE DE DOENÇA VASCULAR CEREBRAL
}

\author{
MARCIA MAIUMI FUKUIMA*, TÂNIA LEME DA ROCHA MARTINEZ* *, \\ LEONOR DO ESPÍRITO SANTO DE ALMEIDA PINTO* *, CAIO R.C. AURIEMO**, \\ LUIZAUGUSTO FRANCO DE ANDRADE*
}

\begin{abstract}
RESUMO - No protocolo de avaliaçāo clínico-laboratorial de pacientes com acidente vascular cerebral (AVC) aterotrombótico dosamos $\mathrm{e}$ analisamos níveis de fibrinogênio plasmático (técnica de Clauss automatizada), para determinar seu possível papel como fator de risco trombogênico em 29 pacientes (20 homens e 9 mulheres) com idades entre 25 a 79 anos (mediana=55); todos tinham tido AVC aterotrombótico. Eles foram classificados em 2 grupos segundo alterações de fluxo nas carótidas: gl - sem alteração de fluxo $(n=19)$ e g2- com alteração de fluxo ( $n=10$ ). Resultados- A média das dosagens de fibrinogênio no g1 foi de 269 e no g2 de $353 \mathrm{mg} / \mathrm{d} 1$. Quarenta e sete por cento dos pacientes do gl e $80 \%$ do g2, apresentaram medidas $>300 \mathrm{mg} / \mathrm{dl}$. As diferenças obtidas entre os grupos neste estudo foram significantes. Conclusãa- Considerando o nível de risco epidemiológico de 300 $\mathrm{mg} / \mathrm{dl}$, nossos resultados sugerem que o fibrinogênio é um fator de risco independente para AVC aterotrombótico, especialmente naqueles com alteração de fluxo carotídeo.
\end{abstract}

PALAVRAS CHAVE: doença cerebrovascular, fibrinogênio, fator de risco, ultra-sonografia carotídea.

\section{Fibrinogen as independent risk factor for ischemic stroke}

ABSTRACT - We have studied fibrinogen levels (Clauss technique) in atherothrombotic ischemic stroke patients, in order to determine its role as a thombogenic risk factor. Twenty nine patients ( 20 men and 9 women) between 25 and 79 years old were studied; they all have had a atherothrombotic stroke. They were classified into two groups according to the result of their carotid doppler ultrasonography: $g 1$ - without carotid flow reduction ( $n=19$ ) and $\mathrm{g} 2$ - with carotid flow reduction $(\mathrm{n}=10)$. The fibrinogen mean value was $269 \mathrm{mg} / \mathrm{dl}$ in $\mathrm{g} 1$ and $353 \mathrm{mg} / \mathrm{dl}$ in $\mathrm{g} 2$. There were $47 \%$ of patients in $\mathrm{g} 1$ and $80 \%$ of patients in $\mathrm{g} 2$ who presented levels $>300 \mathrm{mg} / \mathrm{dl}$. The proportions of the groups were significantly different $(p<0,05)$. Considering the epidemiological value of $300 \mathrm{mg} / \mathrm{dl}$, we conclude that the fibrinogen can be an independent risk factor for ischemic atherothrombotic stroke, specially in those whose carotid flow is reduced.

KEY WORDS: stroke, cerebrovascular disease, fibrinogen, risk factor, carotid ultrasonography.

São vários os fatores de risco bem estabelecidos para doenças aterotrombóticas (coronariana e cerebrovascular, principalmente), como hipertensão arterial, hipercolesterolemia e tabagismo, por exemplo. Alguns outros fatores vêm sendo estudados e ainda não há consenso quanto à preditividade de alguns destes para tais eventos vasculares, dentre os quais o fibrinogênio tem se destacado. Há fatores que podem prognosticar maior risco, sendo considerados independentes; estes fatores de risco independentes interferem pela sua magnitude, isto é, quanto maior o seu valor, maior a probabilidade futura de doença.

Estudo realizado na Escola Paulista de Medicina - Universidade Federal de São Paulo (EPM-UNIFESP) *Disciplina de Neurologia, **Setor de Lípides do Departamento de Medicina. Aceite: 7-julho-1997. 
O fibrinogênio é uma glicoproteína com peso molecular de 340000 daltons. Sua molécula contém uma estrutura dimérica simétrica constituída de três pares de cadeias polipeptídicas (cadeia alfa, beta e gama), ligadas entre si por pontes dissulfídricas. É produzida no fígado e acredita-se que seus níveis plasmáticos são regulados através de mecanismo de "feedback" dos seus produtos de degradação e citoquinas produzidas por macrófagos ${ }^{1,3}$. Uma de suas funções está ligada ao processo de coagulação do sangue: este inicia-se por uma lesāo, que consiste de dano funcional e mecânico ao endotélio vascular, seguida de processo inflamatório endotelial e processo degenerativo. A conversão do fibrinogênio em fibrina pela trombina, que é uma protease, é a fase final do processo de coagulação. A fibrina, por sua vez, leva diretamente à agregação plaquetária. $O$ agregado plaquetário, juntamente com a fibrina, formam o coágulo. A cascata de coagulação é um sistema amplificador, no qual os fatores de coagulação XII, X, IX, VII e II têm função enzimática e o fibrinogênio funciona como substrato. Além desta função na coagulação e agregaçāo plaquetária, o fibrinogênio é uma proteína de fase aguda, influi na determinaçāo da viscosidade do sangue e estimula a migração e proliferação de células musculares lisas. Portanto, o aumento dos seus níveis está relacionado a trombose. No entanto, a demonstração da presença de fibrinogênio na camada íntima dos vasos cerebrais sugere que o depósito de fibrinogênio pode preceder o espessamento da camada íntima com posterior formação da placa aterosclerótica ${ }^{15}$. Apesar das evidências da relaçāo entre trombose e aterogênese, 0 mecanismo fisiopatológico exato pelo qual o fibrinogênio participa desses processos permanece incerto ${ }^{3.12}$.

Baseados na ação pró-trombótica do fibrinogênio, elaboramos este estudo para analisar os níveis de fibrinogênio nas alterações do fluxo carotídeo em pacientes com acidente vascular cerebral isquêmico (AVCI) aterotrombótico.

\section{MATERIAL E MÉTODOS}

Foram estudados 29 pacientes com AVCI aterotrombótico com idade entre 25 e 79 anos (mediana=55), sendo 20 do sexo masculino e 9 feminino, atendidos no Setor de Doenças Neurovasculares da Disciplina de Neurologia da EPM-UNIFESP, no período de 1993 a 1996. Todos foram submetidos ao protocolo de investigação etiológica do AVCI, e foram afastadas causas emboligênicas cardíacas.

Os diagnósticos de AVCI foram feitos por quadro clínico e tomográfico e classificados segundo a Classificação de Doenças Cerebrovasculares III, de 1990, do National Institute of Neurological Disorders and Stroke ${ }^{18}$.

Os pacientes foram classificados em dois grupos segundo a presença de alteração de fluxo no doppler de carótidas: grupo $1(\mathrm{gl})=$ sem alteração de fluxo carotídeo $(\mathrm{n}=19$, sendo 8 mulheres e 11 homens) e grupo 2 (g2)= com alteração de fluxo carotídeo $(n=10$, sendo 1 mulher e 9 homens), isto é, com estenose ou obstrução da artéria carótida interna uni ou bilateralmente.

O fibrinogênio foi dosado pela técnica de Clauss automatizada com intervalo inferior a 4 horas entre a coleta e o processamento. $O$ valor de corte arbitrado foi de $300 \mathrm{mg} / \mathrm{dl}$, com base nos estudos epidemiológicos de Framingham, Northwick Park Heart Study e PROCAM. ${ }^{8,11,13}$ Todos os pacientes tiveram a amostra sanguínea colhida no mínimo 60 dias após o AVC, sem nenhum processo inflamatório ou infeccioso vigente. Todos os tabagistas haviam abandonado o hábito na ocasião da instalação do AVC.

Para a análise estatística foi utilizado o teste de Mann-Whitney, com significância de $\mathrm{p}<0,05$ e foram assinalados com $\left(^{*}\right)$ os valores significantes.

\section{RESULTADOS}

A média das dosagens no grupo 1 foi $269 \mathrm{mg} / \mathrm{dl}$ e no grupo $2,353 \mathrm{mg} / \mathrm{dl}$ ( $\mathrm{gl}<\mathrm{g} 2^{*}$ ). Observouse na populaçāo como um todo $59 \%$ dos valores acima de $300 \mathrm{mg} / \mathrm{dl}$ e no grupo $2,80 \%$ acima deste nivel. Os valores individuais são mostrados na Figura 1.

\section{DISCUSSÃO}

O nível plasmático de fibrinogênio é de 1,5 a 4,5 g/l, definido pela curva normal da população. Para manter a hemostase necessita-se de concentração mínima de $0,5 \mathrm{~g} / \mathrm{l}$. Esse nível é geneticamente 


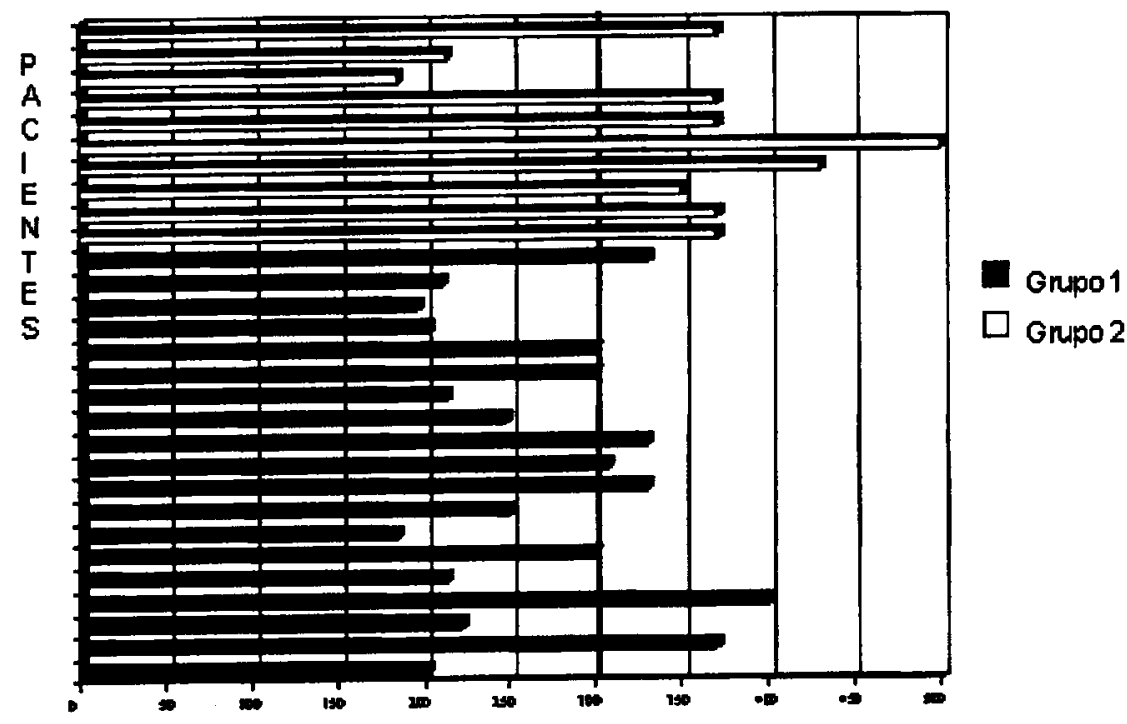

Fibrinogetrio [ majd] ]

Fig l. Fibrinogênio em mg/dl dos pacientes do grupo 1 (sem alteração de fluxo carotideo) e do grupo 2 (com alteração de fluxo carotideo).

definido em cerca de $50 \% \%^{7.9}$. A determinação genética do fibrinogênio é dependente do polimorfismo do DNA do gene do $\beta$-fibrinogênio. Cerca de $6 \%$ da variação individual dos niveis de fibrinogênio se deve ao tabagismo, $3 \%$ à idade e massa corporal ${ }^{10}$. Outros fatores ambientais que contribuem para a variação do fibrinogênio são diabetes melito, hipercolesterolemia, hipertrigliceridemia, hipertensão arterial, estresse psicossocial, menopausa e anticoncepcional oral. Outra parcela varia em decorrência de reações inflamatórias agudas e crônicas, sendo a interleucina- 6 o mediador da reação inflamatória. Grandes estudos epidemiológicos consideraram níveis de fibrinogênio de 2,86 a 3,56 g/1 (PROCAM e Göteborg) ${ }^{8,17}$ como de alto risco para doenças cardiovasculares, mostrando o estudo de Framingham aumento significante de eventos cardiovasculares para concentrações acima de $3,0 \mathrm{~g} / 1^{11}$.

Sabe-se que os níveis de fibrinogênio aumentam na instalação do AVC, como reação de fase aguda, devido à necrose do tecido cerebral ${ }^{14,16}$. Altos níveis de fibrinogênio também já foram considerados de valor prognóstico, já que a hiperfibrinogenemia, pelo efeito hemorreológico, pode limitar a perfusāo cerebral 4 . Porém, como a viscosidade sanguínea e a agregabilidade eritrocitária estão aumentadas nesses pacientes com AVCI, há evidências de que os níveis de fibrinogênio estivessem previamente elevados ${ }^{2,35,6}$. $\mathrm{O}$ fibrinogênio foi considerado importante fator de risco independente, em casos de AVC, nos estudos de Framingham e Göteborg ${ }^{16.17}$.

Na nossa amostra, constituída de pacientes que já sofreram pelo menos um evento cerebrovascular, 59\% apresentaram níveis de fibrinogênio acima dos considerados como risco para eventos cardiovasculares por estudos epidemiológicos realizados na América do Norte e Europa. A diferença significante entre os grupos com e sem redução de fluxo carotídeo sugere que o fibrinogênio pode representar um fator de risco independente para AVC aterotrombótico em pacientes com alteração do fluxo carotídeo. Considerando-se nossos achados, pacientes sintomáticos e assintomáticos com alteração do fluxo carotídeo associado a hiperfibrinogenemia poderiam ser alvo de algumas medidas gerais (alterando hábitos) e medicamentosas para redução dos niveis de fíbrinogênio, visando à 
prevençāo de eventos vasculares. Faltam ainda estudos epidemiológicos no nosso meio para permitir conclusões mais seguras e apuradas. Novos estudos devem ser realizados com controle e amostras maiores no futuro.

Agradecimentos - Agradecemos ao Prof. Dr. Neil Ferreira Novo e à Profa. Dra. Yara Juliano pela orientação da análise estatística.

\section{REFERENCIAS}

1. Cook NS, Ubben D. Fïbrinogen as a major risk in cardiovascular disease. TIPS 1990;11: 444-451.

2. Ernst E, Matrai A, Marshall M. Blood rheology in patients with transient ischemic atacks. Stroke 1988;19: 624-636.

3. Ernst E, Resch KL. The "fibrinogen hypothesis"; is elevated plasma fibrinogen a major cardiovascular risk factor? In Woodford FP, Davignon J, Sniderman A (eds). Atherosclerosis X. Amsterdam: Elsevier, 1995:785-792.

4. Ernst E, Resch KL, Matrai A, Buhl M, Schlosser P, Paulsen HF. Impaired blood rheology: a risk factor after stroke? J Intern Med 1991;229:457-462.

5. Fletcher AP, Alkjaersig N, Davis A, Lewis M. Blood coagulation and plasma fibrinolitic enzyme system pathophysiology in stroke. Stroke 1976;7:337-348.

6. Grotta J, Ackerman R, Comeia J, Fallick G, Chang J. Whole blood viscosity parameters and cerebral blood flow. Stroke 1982;13:296-301.

7. Hamsten A, Iselius L, de Faire U, Blombäck M. Genetic and cultural inheritance of plasma fibrinogen concentration. Lancet 1987;2:988-990.

8. Heinrich J, Schulte H, Balleisen L, Assmann G, van de Loo J. Predictive value of haemostatic variables in the PROCAMstudy. Thromb Haemostas 1991;65:815.

9. Humphries SE, Cook M, Dubowitz M, Stirling Y, Meade TW. Role of genetic variation at the fibrinogen locus in delermination of plasma fibrinogen concentrations. Lancet 1987,1:1452-1454.

10. Kannel WB, D'Agostinho RB, Belanger AJ. Fibrinogen, cigarette smoking, and risk of cardiovascular disease: insights from The Framingham Study. Am Heart J 1987;113:1006-1010.

11. Kannel WB, Wolf PA, Castelli WP, D'Agostinho RB. Fibrinogen and risk of cardiovascular disease. JAMA 1987;258:1183-1186.

12. Mann KG. Blood coagulation: linkage between thombosis and atherosclerosis: an overview. In Woodford FP, Davignon J, Sniderman A (eds). Atherosclerosis X. Amsterdam; Elsevier, 1995:780-784,

13. Meade TW, Brozovic M, Chakrabarti RR, et al.. Haemostatic funcion and ischaemic heart disease: principal results of the Northwick Park Heart Study. Lancet 1986;2:533-537.

14. Pilgeram LO, Chee AN, von dem Bussche G. Evidence for abnormalities in clotting and thrombolysis as a risk factor for stroke. Stroke 1973;4:643-657.

15. Sadoshima S, Tanaka K. Fibrinogen and low density lipoprotein in the development of cerebral atherosclerosis. Atherosclerosis 1979;34:93-103.

16. Todd M, McDevitt E, McDowell F. Stroke and blood coagulation. Stroke 1973;4:400-405.

17. Wilhelmsen L, Svärdsudd K, Korsan-Bengtsen K, Larsson B, Welin L, Tibblin G. Fibrinogen as a risk factor for stroke and myocardial infarction. N Engl J Med 1984;311:501-505.

18. Whisnant JP, Basford JR, Bernstein EF et al. Special report from the National Institute of Neurological Disorders and Stroke: Classification of Cerebrovascular Disease III. Stroke 1990;21:637-676. 